\title{
Positive Association between ANKRD55 Polymorphism 7731626 and Dermatomyositis/Polymyositis with Interstitial Lung Disease in Chinese Han Population
}

\author{
Liubing Li, ${ }^{1}$ Si Chen, ${ }^{1,2}$ Xiaoting Wen, ${ }^{1}$ Qian Wang,, Guanting Lv, ${ }^{1,3}$ Jing Li, \\ Funing Yang, ${ }^{1,4}$ Fengchun Zhang, ${ }^{1}$ and Yongzhe $\mathrm{Li}^{1}$ \\ ${ }^{1}$ Department of Rheumatology and Clinical Immunology, Peking Union Medical College Hospital, Chinese Academy of \\ Medical Sciences \& Peking Union Medical College, Key Laboratory of Rheumatology and Clinical Immunology, Ministry of Education, \\ Beijing, China \\ ${ }^{2}$ Department of Clinical Laboratory, Beijing Anzhen Hospital, Capital Medical University, Beijing, China \\ ${ }^{3}$ Department of Blood Transfusion, Tangdu Hospital, The Fourth Military Medical University, Xian, China \\ ${ }^{4}$ Department of Medical Laboratory, The First Hospital of Jilin University, Changchun, China
}

Correspondence should be addressed to Yongzhe Li; yongzhelipumch@126.com

Received 13 January 2017; Revised 17 March 2017; Accepted 21 March 2017; Published 2 April 2017

Academic Editor: Dimitrios P. Bogdanos

Copyright (C) 2017 Liubing Li et al. This is an open access article distributed under the Creative Commons Attribution License, which permits unrestricted use, distribution, and reproduction in any medium, provided the original work is properly cited.

Single nucleotide polymorphisms (SNPs) in TNFSF4 and ANKRD55 genes have been shown to be associated with several autoimmune diseases, although whether these genes are susceptibility genes for dermatomyositis/polymyositis (DM/PM) has, to date, not been reported. This study aimed to investigate the potential associations of these SNPs with DM/PM in a Chinese Han population. Five SNPs in TNFSF4 (rs2205960, rs844644, and rs844648) and ANKRD55 (rs6859219, rs7731626) genes were genotyped using the SequenomMassArray system in 2297 Chinese individuals. In total, 1017 DM/PM patients and 1280 gendermatched healthy controls were genotyped. No significant associations were observed in DM/PM patients for the five SNPs analyzed. The association between SNPs and interstitial lung disease (ILD) was also investigated. Both DM-ILD $\left(P_{c}=0.030, \mathrm{OR}=0.65,95 \%\right.$ CI: $0.47-0.88)$ and DM/PM-ILD $\left(P_{c}=0.015, \mathrm{OR}=0.67,95 \% \mathrm{CI}\right.$ : $\left.0.51-0.87\right)$ exhibited a significant association with the rs7731626-A allele. Rs7731626-A was less frequently found in DM-ILD and DM/PM-ILD patients compared with healthy controls. This is the first study to demonstrate a positive association between ANKRD55 polymorphism and DM-ILD and DM/PM-ILD. A decreased frequency of rs7731626-A in DM-ILD and DM/PM-ILD patients suggests that the A variant may be protective against DM/PM-ILD.

\section{Introduction}

Dermatomyositis (DM) and polymyositis (PM) are autoimmune diseases that are most prevalent in women and the elderly [1], characterized by the shared features of skeletal muscle weakness, elevated serum creatine kinase levels, and inflammation in muscle biopsy [2, 3]. It has been reported that DM/PM prevalence ranges from 21.42 to $32.74 / 100,000$ person-years (py) in the USA $[1,4,5]$ and from 10 to $13 / 100,000$ py in Japan with an increasing trend [6]. Though $\mathrm{DM} / \mathrm{PM}$ most frequently affects the skin and muscles, it may also affect multiple organs, most notably, the lung. Interstitial lung disease (ILD) is the primary pulmonary mani- festation, which is associated with a high morbidity and mortality in DM/PM patients [7-9]. It would, therefore, be advantageous to investigate the biomarkers associated with the development of ILD in patients with DM/PM. To date, several genetic [10-12] and environmental [13-16] factors have been proposed to contribute to the development of DM/PM. However, these identified predisposing factors are unable to completely account for the pathogenesis of $\mathrm{DM} / \mathrm{PM}$.

Studies have reported that autoimmune diseases share a number of human leukocyte antigen (HLA) and non-HLA genes [12, 17-19]. A genome-wide association study (GWAS) revealed DM shares genetic susceptibility factors with 
rheumatoid arthritis (RA), multiple sclerosis (MS), systemic lupus erythematosus (SLE), and other autoimmune diseases [12]. Recent studies identified that single nucleotide polymorphisms (SNPs) of TNFSF4 (rs2205960, rs844644, and rs844648) and ANKRD55 (rs6859219, rs7731626) also shared susceptibility loci for RA [20-22], MS [17], and SLE $[23,24]$. The association of the five SNPs with the diseases discussed is summarized in Table S1 (see Table S1 in the Supplementary Material available online at https://doi.org/10.1155/ 2017/2905987). TNFSF4 encodes the ligand for TNFRSF4, and this receptor-ligand pair can activate CD4+ T cells [25]. The TNFSF4-TNFRSF4 interaction has been shown to contribute to the induction of antitumor immunity [26] and the inhibition of allergic responses [27, 28]. ANKRD55 encodes ankyrin repeat domain-containing protein 55 , which mediates protein-protein interactions. A recent report has revealed that $A N K R D 55$ can be detected in resting $C D 4+\mathrm{T}$ cells and monocytes and may have possible relevance to autoimmune diseases (http://www.amazonia.transcriptome.eu) [29]. However, the exact function of ANKRD55 remains unknown. In order to elucidate whether the five genetic polymorphisms of TNFSF4 and ANKRD55 are associated with DM/PM in a Chinese Han population, we performed the current study including 1017 DM/PM patients and 1280 matched healthy controls.

\section{Materials and Methods}

2.1. Subjects. The current study was a multicenter casecontrol study, approved by the Ethics Committee of the Peking Union Medical College Hospital, China. Patients with DM/PM fulfilled the Bohan and Peter criteria [30] and were recruited between February 2013 and June 2015. A total of $1017 \mathrm{DM} / \mathrm{PM}$ patients including 569 patients from the Peking Union Medical College Hospital, along with 448 patients from another 21 centers in China, were enrolled. The sera from 659 recruited patients were analyzed for antiJo-1 antibody using QUANTA Lite Jo-1-ELISA assay (Inova Diagnostics, San Diego, CA, USA). Patients with ILD were identified with high-resolution chest computed tomography (HRCT). In addition, 1280 gender-matched healthy individuals from the Peking Union Medical College Hospital were recruited in this study. $287 \mathrm{DM} / \mathrm{PM}$ patients from Peking Union Medical College Hospital were followed up for 3 yrs. All participants were from the Chinese Han population and provided signed informed consent.

2.2. Genotyping. We collected $2 \mathrm{ml}$ peripheral blood from each participant in an ethylenediaminetetraacetic acid(EDTA-) coated tube. DNA was extracted from peripheral white blood cell using genomic DNA kits (Tiangen, Beijing, China) and stored at $-80^{\circ} \mathrm{C}$ until use. The genotyping of the five SNPs from TNFSF4 and ANKRD55 genes was performed using SequenomMassArray system (San Diego, CA, USA) according to the manufacturer's protocol. Primers for polymerase chain reaction (PCR) and locus-specific single-base extension were designed by MassArray Assay Design 4.0 (Sequenom). Briefly, all DNA samples were transferred to a 384-well plate. After multiplex PCR amplications, the products were used for locus-specific single-base extension reaction. Afterwards, the products were desalted and transferred onto a 384-element SpectroCHIParray (Sequenom). Allele detection was conducted by matrixassisted laser desorption/ionization-time-of-flight mass spectrometry (MALDI-TOF MS). Finally, MassArrayTyper 4.0 software was used to analyze the resultant mass spectrograms and genotype data.

2.3. Statistical Analysis. The Chi-square $\left(\chi^{2}\right)$ test was used to assess whether each SNP in the control populations conformed to Hardy-Weinberg equilibrium (HWE). Any SNPs that deviated from the HWE $(P<0.05$ in the controls $)$ would be excluded in the analysis. The differences in allelic and genotypic frequencies between patients and controls were calculated by $\chi^{2}$ test. The risk allele frequency for each SNP between DM/PM patients with ILD and controls was calculated. The odds ratio (OR) and 95\% confidence interval $(95 \% \mathrm{CI})$ of the association were calculated; $P$ values $<0.05$ (adjusted by Bonferroni correction) were considered to be statistically significant. The logistic regression models (additive, dominant, and recessive models) were used to further analyze the genotype frequencies. For the association analysis between the five SNPs and the three subsets (DM, PM, or overall $\mathrm{DM} / \mathrm{PM}$ versus controls), statistical analysis was performed using PLINK v1.07 (Shaun Purcell, Boston, MA, USA) [31]. The association study for the five SNPs and the presence of ILD were performed according to the results of the following comparisons: patients with ILD versus patients without ILD, patients with ILD versus controls, and patients without ILD versus controls.

\section{Results}

3.1. Clinical Characteristics of Participants. The demographics and clinical features of enrolled DM/PM patients and healthy controls were displayed in Table 1 . A total of 1017 $\mathrm{DM} / \mathrm{PM}$ patients (25.7\% male, $74.3 \%$ female; mean age $46.1 \pm$ 15.2) were recruited, including $654 \mathrm{DM}$ patients and $363 \mathrm{PM}$ patients. Among these patients, 390 of 654 patients had DM and 195 of 363 patients had PM complicated by ILD. In total, 585 of 1017 patients had DM/PM complicated by ILD, while 432 did not. Of $659 \mathrm{DM} / \mathrm{PM}$ patients who were assessed for anti-Jo-1 antibody, 115 patients (61 DM patients and 54 PM patients) were positive for anti-Jo-1 antibody. During the 3year follow-up, $14 \mathrm{DM} / \mathrm{PM}$ patients developed new malignant tumors, including six patients with lung cancer, three with breast cancer, two with ovarian cancer, one with liver cancer, one with nasopharynx cancer, and one with synovial sarcoma. The analysis of the five selected SNPs is shown in Table 2. All SNPs had call rates $>99 \%$ and followed HWE in the controls $\left(P_{c}>0.05\right.$, Table 2$)$.

3.2. Association of the Five SNPs with DM, PM, or DM/PM in the Han Population. Five SNPs rs2205960, rs844644, rs844648, rs6859219, and rs7731626 were genotyped in 1017 $\mathrm{DM} / \mathrm{PM}$ patients (DM, $n=654 ; \mathrm{PM}, n=363)$ and 
TABLE 1: Clinical features of DM/PM patients and healthy controls.

\begin{tabular}{lccc}
\hline & Cases & & Controls \\
& Number/total & $\%$ & Number/total \\
\hline Mean age & $46.1 \pm 15.2$ & & $41.8 \pm 12.7$ \\
Male & $261 / 1017$ & 25.7 & $165 / 1280$ \\
Female & $756 / 1017$ & 74.3 & $1115 / 1280$ \\
DM & $654 / 1017$ & 64.3 & - \\
PM & $363 / 1017$ & 35.7 & - \\
ILD & $585 / 1017$ & 57.5 & - \\
DM with ILD & $390 / 1017$ & 38.3 & - \\
PM with ILD & $195 / 1017$ & 19.2 & - \\
Anti-Jo-1 antibody & $115 / 659$ & 17.5 & - \\
DM with anti-Jo-1 antibody & $61 / 438$ & 13.9 & - \\
PM with anti-Jo-1 antibody & $54 / 221$ & 24.4 & - \\
Malignancy & $14 / 287$ & 4.9 & - \\
Lung cancer & $6 / 14$ & 42.9 & - \\
Breast cancer & $3 / 14$ & 21.4 & - \\
Ovarian cancer & $2 / 14$ & 14.3 & - \\
Liver cancer & $1 / 14$ & 7.1 & - \\
Nasopharynx cancer & $1 / 14$ & 7.1 & - \\
Synovial sarcoma & $1 / 14$ & 7.1 & - \\
\hline
\end{tabular}

$\mathrm{DM}$ = dermatomyositis; $\mathrm{PM}$ = polymyositis; $\mathrm{ILD}$ = interstitial lung disease.

TABLE 2: The information of five selected SNPs.

\begin{tabular}{lccccccccc}
\hline Gene & SNP & Chromosome & Position & Function class & Allele & $\begin{array}{c}\text { MAF in CHB } \\
\text { (dbSNP) }\end{array}$ & $\begin{array}{c}\text { MAF } \\
P_{c} \text { for HWE }\end{array} \begin{array}{c}\text { Call rate } \\
(\%)\end{array}$ \\
\hline TNFSF4 & rs2205960 & 1 & 173222336 & NA & G > T & 0.198 & 0.265 & 0.655 \\
TNFSF4 & rs844644 & 1 & 173240356 & Intron & $\mathrm{A}>\mathrm{C}$ & 0.384 & 0.445 & NS & 99.96 \\
TNFSF4 & rs844648 & 1 & 173254724 & Intron & G > A & 0.384 & 0.453 & NS & 100 \\
ANKRD55 & rs6859219 & 5 & 56142753 & Intron & C > A & 0.012 & 0.005 & NS & 100 \\
ANKRD55 & rs7731626 & 5 & 56148856 & Intron & G > A & NA & 0.089 & NS & 100 \\
\hline
\end{tabular}

The information was gathered from dbSNP database in NCBI. SNPs = single nucleotide polymorphisms; MAF = minor allele frequency; CHB = Han Chinese in Beijing, China; $P_{c}=P$ value corrected by Bonferroni method; $\mathrm{HWE}=$ Hardy-Weinberg equilibrium; NA = not available; NS = not significant.

1280 normal controls. The allelic and genotypic frequency distribution of the five SNPs are summarized in Table 3. No significant difference in allelic and genotypic frequencies was found between patients and controls $\left(P_{c}>0.05\right.$, Table 3$)$. We further performed logistic regression analysis based on three genetic models (additive, dominant, and recessive models). None of the three genetic models showed any significant difference between patients and controls for the five SNPs $\left(P_{c}>0.05\right.$, Table 4$)$.

3.3. Association between the Five SNPs and ILD Phenotype of $D M / P M$. To analyze the association between the five SNPs and ILD phenotype of DM/PM patients, the five SNPs of TNFSF4 and ANKRD55 were genotyped in $585 \mathrm{DM} / \mathrm{PM}$ patients with ILD (DM-ILD, $n=390$; PM-ILD, $n=195$ ). A significantly decreased frequency of SNP rs7731626-A was observed in DM-ILD (6.54\% in DM-ILD, 9.77\% in the controls, $P_{c}=0.030, \mathrm{OR}=0.65,95 \%$ CI: $0.47-0.88$, Table 5$)$ and DM/PM-ILD patients (6.75\% versus $9.77 \%, P_{c}=0.015$, $\mathrm{OR}=0.67,95 \% \mathrm{CI}: 0.51-0.87$, Table 5$)$ as compared to healthy controls. No significant association was observed between SNP rs7731626-A and PM patients with ILD. The clinical significance of SNP rs7731626-A for DM-ILD and DM/PMILD still needs further study. In addition, no significant association was detected between the other four SNPs and patients with ILD or without ILD when comparing patients with ILD versus patients without ILD, patients with ILD versus controls, or patients without ILD versus controls $\left(P_{c}>\right.$ 0.05 , Table 5).

\section{Discussion}

In this multiple-center, large-sample case-control study, we investigated the associations of TNFSF4 (rs2205960, rs844644, and rs844648) and ANKRD55 (rs6859219, rs7731626) polymorphisms with the susceptibility to DM/PM in a Chinese Han population. Our results demonstrated that ANKRD55 polymorphism (rs7731626) was significantly associated with DM-ILD as well as DM/PM-ILD. A significant decrease in the frequency of rs7731626-A allele in 


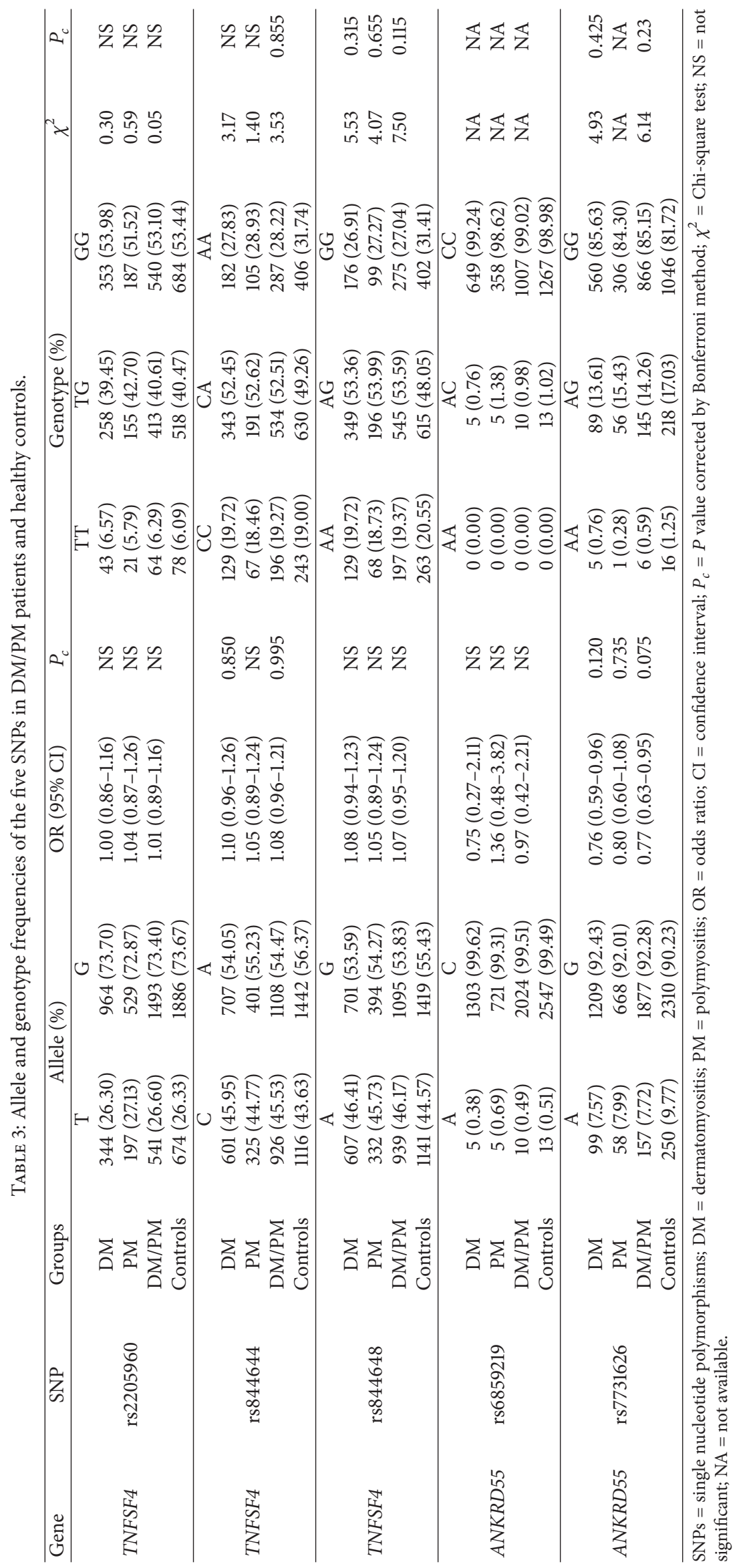




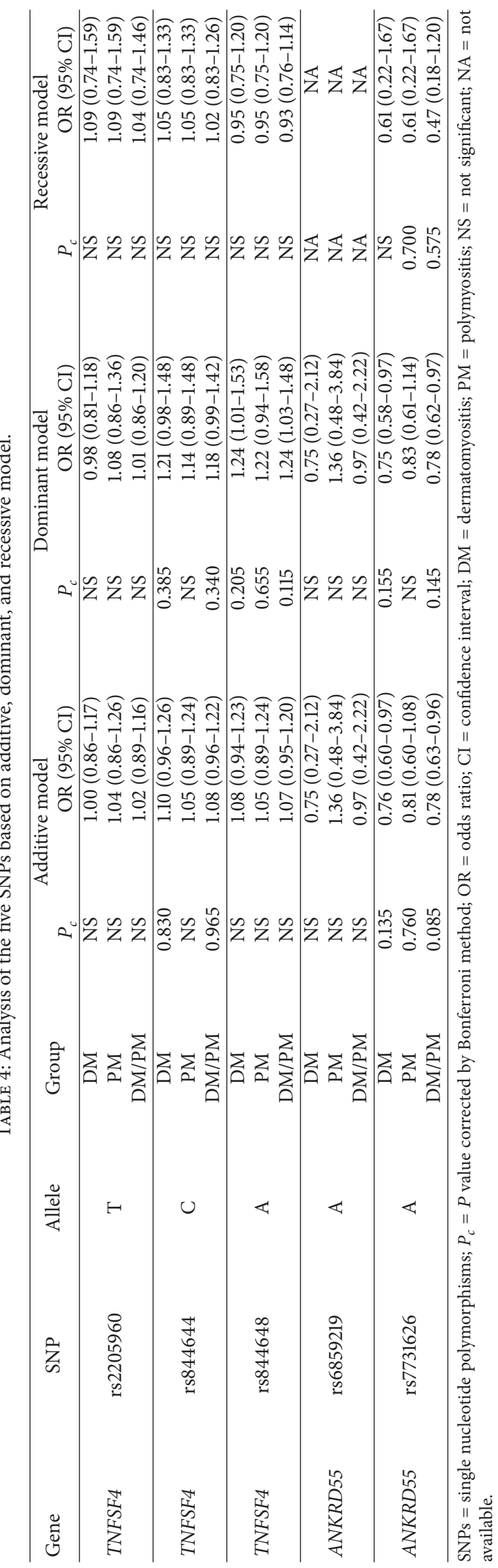




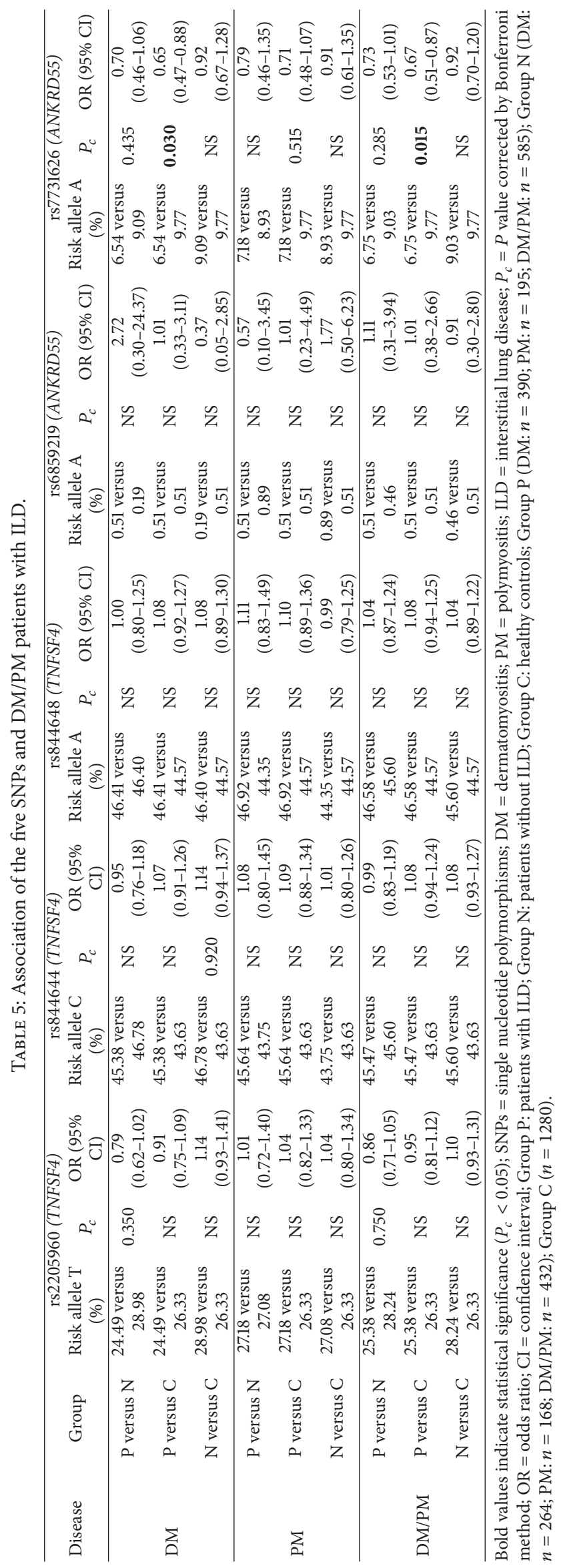


DM-ILD and DM/PM-ILD patients compared to controls may suggest that this allele may play a protective role against the development of ILD. Notably, this was the first study to demonstrate that the ANKRD55 polymorphism was associated with Chinese DM/PM patients with ILD.

ILD is a common complication of DM/PM and progression of ILD is a leading course of mortality [7-9]. Several gene polymorphisms have been revealed to be associated with DM/PM patients with ILD, including STAT4 rs7574865 polymorphism [32] and TNFAIP3 rs2230926 and rs5029939 polymorphisms [33]. However, these identified susceptibility genes do not fully account for the genetic pathogenesis of DM/PM-ILD. ANKRD55 has been shown to be inducible following inflammatory stimuli, and its expression may also increase susceptibility to inflammation in patients [29]. An increase in the protein expression of ANKRD55 in autoimmune encephalomyelitis mice suggests that this molecule may act as a disease biomarker. Although the precise function of ANKRD55 is still unknown, a link between genetic variants of ANKRD55 and autoimmune diseases may exist. At loci ANKRD55, rs7731626 is located in immune cell enhancer regions [34]. To date, few studies have examined the association of SNP rs7731626 of ANKRD55 with different diseases. Okada et al. reported that rs7731626-G was a risk factor for the development of RA by comparing 12,841 RA patients with 33,416 healthy controls in a European population [20]. In our presented study, our data demonstrated rs7731626 was negatively associated with DM-ILD and DM/PM-ILD in Chinese Han patients, whereby the protective A allele was the minor allele in these patients. Our results indicated that DM/PM-ILD may share a common genetic locus with RA. However, no association was found between DM/PM and SNP rs6859219 of ANKRD55, which has been shown to be related to MS [17, 35] and RA [21, 22] (Table S1).

TNFSF4 with its receptor TNFRSF4 promotes CD4+ T cells activation as a potent costimulatory signal [36]. Recent studies have demonstrated that polymorphisms of TNFSF4 (rs2205960, rs844644, and rs844648) are associated with SLE $[23,24,37,38]$ and systemic sclerosis (SSc) [39] (Table S1). To explore whether SNPs rs2205960, rs844644, and rs844648 are also associated with $\mathrm{DM} / \mathrm{PM}$, we conducted the present study. Our data showed no statistically significant association existed between TNFSF4 polymorphisms and DM/PM, suggesting the pathogenesis of DM/PM may differ from SLE and SSc. Additional studies in different ethnic groups should be performed in the future to further investigate the association of rs2205960, rs844644, and rs844648 with DM/PM.

There are, however, some limitations to our study. The current case-control study revealed that the minor allele (A) of rs7731626 was less frequent in DM-ILD and DM/PM-ILD than in controls (Table 5). We hypothesize that the A variant may be protective for DM/PM-ILD. However, in order to decide if this result is robust enough to also be clinically significant, multiple studies with different sample sizes are needed in the future study. Whether this variant affects the properties of ANKRD55 protein in a way which is compatible with this hypothesis requires further confirmation. In addition, we only investigated the association of rs7731626 with DM-ILD or DM/PM-ILD in the Han Chinese population.
Due to ethnic genetic differences, further investigations are required to confirm the association of ANKRD55 genetic variants with DM-ILD or DM/PM-ILD in Caucasian and African patients.

\section{Conclusions}

This study was the first study to elucidate that a rare variant within the ANKRD55 gene (rs7731626) is protective in DMILD and DM/PM-ILD in a Chinese Han population. Further studies are needed to investigate the association of ANKRD55 genetic variants with DM-ILD or DM/PM-ILD in different ethnic groups from variable geographical regions.

\section{Conflicts of Interest}

The authors declare that there are no conflicts of interest regarding the publication of this paper.

\section{Authors' Contributions}

Liubing Li, Si Chen, and Xiaoting Wen contributed equally to this manuscript.

\section{Acknowledgments}

The authors thank all the participants for their cooperation. They also thank the other hospital staff that helped collect the blood samples and clinic data. This study is supported by funding from the Research Special Fund for Public Welfare Industry of Health (201202004), the National Natural Science Foundation of China Grants $(81172857,81373188)$, the Chinese National High Technology Research and Development Program, Ministry of Science and Technology Grants (2011AA02A113), the National Science Technology Pillar Program in the 12th Five-Year Plan (2014BAI07B00), and the Capital Health Research and Development of Special (2014$1-4011)$.

\section{References}

[1] S. Bernatsky, L. Joseph, C. A. Pineau et al., "Estimating the prevalence of polymyositis and dermatomyositis from administrative data: age, sex and regional differences," Annals of the Rheumatic Diseases, vol. 68, no. 7, pp. 1192-1196, 2009.

[2] J. E. Hoogendijk, A. A. Amato, B. R. Lecky et al., "119th ENMC international workshop: trial design in adult idiopathic inflammatory myopathies, with the exception of inclusion body myositis, 10-12 October 2003, Naarden, The Netherlands," Neuromuscular Disorders, vol. 14, no. 5, pp. 337-345, 2004.

[3] M. C. Dalakas and R. Hohlfeld, "Polymyositis and dermatomyositis," The Lancet, vol. 362, no. 9388, pp. 971-982, 2003.

[4] K. E. Smoyer-Tomic, A. A. Amato, and A. W. Fernandes, "Incidence and prevalence of idiopathic inflammatory myopathies among commercially insured, Medicare supplemental insured, and Medicaid enrolled populations: an administrative claims analysis," BMC Musculoskeletal Disorders, vol. 13, article 103, 2012. 
[5] M. J. Bendewald, D. A. Wetter, X. Li, and M. D. P. Davis, "Incidence of dermatomyositis and clinically amyopathic dermatomyositis: a population-based study in Olmsted County, Minnesota," Archives of Dermatology, vol. 146, no. 1, pp. 26-30, 2010.

[6] A. Ohta, M. Nagai, M. Nishina, H. Tomimitsu, and H. Kohsaka, "Prevalence and incidence of polymyositis and dermatomyositis in Japan," Modern Rheumatology, vol. 24, no. 3, pp. 477-480, 2014.

[7] R. W. Hallowell, D. P. Ascherman, and S. K. Danoff, "Pulmonary manifestations of polymyositis/dermatomyositis," Seminars in Respiratory and Critical Care Medicine, vol. 35, no. 2, pp. 239248, 2014.

[8] I. Marie, P. Y. Hatron, S. Dominique, P. Cherin, L. Mouthon, and J.-F. Menard, "Short-term and long-term outcomes of interstitial lung disease in polymyositis and dermatomyositis: a series of 107 patients," Arthritis and Rheumatism, vol. 63, no. 11, pp. 3439-3447, 2011.

[9] I. Marie, E. Hachulla, P. Chérin et al., "Interstitial lung disease in polymyositis and dermatomyositis," Arthritis Care and Research, vol. 47, no. 6, pp. 614-622, 2002.

[10] C.-E. Zhang, Y. Li, Z.-X. Wang et al., "Variation at HLA-DPB1 is associated with dermatomyositis in Chinese population," Journal of Dermatology, vol. 43, no. 11, pp. 1307-1313, 2016.

[11] F. W. Miller, W. Chen, T. P. O'Hanlon et al., "Genome-wide association study identifies HLA 8.1 ancestral haplotype alleles as major genetic risk factors for myositis phenotypes," Genes and Immunity, vol. 16, no. 7, pp. 470-480, 2015.

[12] F. W. Miller, R. G. Cooper, J. Vencovský et al., "Genome-wide association study of dermatomyositis reveals genetic overlap with other autoimmune disorders," Arthritis and Rheumatism, vol. 65 , no. 12, pp. 3239-3247, 2013.

[13] Z. E. Betteridge, H. Gunawardena, and N. J. McHugh, "Pathogenic mechanisms of disease in myositis: autoantigens as clues," Current Opinion in Rheumatology, vol. 21, no. 6, pp. 604-609, 2009.

[14] T. Klopstock, “Drug-induced myopathies," Current Opinion in Neurology, vol. 21, no. 5, pp. 590-595, 2008.

[15] S. Okada, E. Weatherhead, I. N. Targoff, R. Wesley, and F. W. Miller, "Global surface ultraviolet radiation intensity may modulate the clinical and immunologic expression of autoimmune muscle disease," Arthritis and Rheumatism, vol. 48, no. 8, pp. 2285-2293, 2003.

[16] J. Cukier, R. A. Beauchamp, J. S. Spindler, S. Spindler, C. Lorenzo, and D. E. Trentham, "Association between bovine collagen dermal implants and a dermatomyositis or a polymyositis-like syndrome," Annals of Internal Medicine, vol. 118, no. 12, pp. 920-928, 1993.

[17] C. M. Lill, B.-M. M. Schjeide, C. Graetz et al., "Genome-wide significant association of ANKRD55 rs6859219 and multiple sclerosis risk," Journal of Medical Genetics, vol. 50, no. 3, pp. 140143, 2013.

[18] C. Terao, K. Ohmura, Y. Kawaguchi et al., "PLD4 as a novel susceptibility gene for systemic sclerosis in a Japanese population," Arthritis and Rheumatism, vol. 65, no. 2, pp. 472-480, 2013.

[19] C. Miceli-Richard, E. Comets, P. Loiseau, X. Puechal, E. Hachulla, and X. Mariette, "Association of an IRF5 gene functional polymorphism with Sjögren's syndrome," Arthritis and Rheumatism, vol. 56, no. 12, pp. 3989-3994, 2007.

[20] Y. Okada, D. Wu, G. Trynka et al., "Genetics of rheumatoid arthritis contributes to biology and drug discovery," Nature, vol. 506, no. 7488, pp. 376-381, 2014.
[21] A. Zhernakova, E. A. Stahl, G. Trynka et al., "Meta-analysis of genome-wide association studies in celiac disease and rheumatoid arthritis identifies fourteen non-HLA shared loci," PLoS Genetics, vol. 7, no. 2, Article ID e1002004, 2011.

[22] E. A. Stahl, S. Raychaudhuri, E. F. Remmers et al., "Genomewide association study meta-analysis identifies seven new rheumatoid arthritis risk loci," Nature Genetics, vol. 42, no. 6, pp. 508-514, 2010.

[23] H. Manku, C. D. Langefeld, S. G. Guerra et al., “Trans-ancestral studies fine map the SLE-susceptibility locus TNFSF4," PLoS Genetics, vol. 9, no. 7, Article ID e1003554, 2013.

[24] X.-J. Zhou, X.-L. Lu, S. K. Nath et al., "Gene-gene interaction of BLK, TNFSF4, TRAF1, TNFAIP3, and REL in systemic lupus erythematosus," Arthritis and Rheumatism, vol. 64, no. 1, pp. 222-231, 2012.

[25] P. R. Baum, R. B. Gayle III, F. Ramsdell et al., "Molecular characterization of murine and human OX40/OX40 ligand systems: identification of a human OX40 ligand as the HTLV-1regulated protein gp34," EMBO Journal, vol. 13, no. 17, pp. 39924001, 1994.

[26] J. Zaini, S. Andarini, M. Tahara et al., "OX40 ligand expressed by DCs costimulates NKT and CD4+ Th cell antitumor immunity in mice," Journal of Clinical Investigation, vol. 117, no. 11, pp. 3330-3338, 2007.

[27] G. Gri, S. Piconese, B. Frossi et al., "CD4+CD25+ regulatory $\mathrm{T}$ cells suppress mast cell degranulation and allergic responses through OX40-OX40L interaction," Immunity, vol. 29, no. 5, pp. 771-781, 2008.

[28] D. Seshasayee, W. P. Lee, M. Zhou et al., "In vivo blockade of OX40 ligand inhibits thymic stromal lymphopoietin driven atopic inflammation," Journal of Clinical Investigation, vol. 117, no. 12, pp. 3868-3878, 2007.

[29] A. L. de Lapuente, A. Feliu, N. Ugidos et al., "Novel insights into the multiple sclerosis risk gene ANKRD55," The Journal of Immunology, vol. 196, no. 11, pp. 4553-4565, 2016.

[30] A. Bohan and J. B. Peter, "Polymyositis and dermatomyositis I," New England Journal of Medicine, vol. 292, no. 7, pp. 344-347, 1975.

[31] A. D. Skol, L. J. Scott, G. R. Abecasis, and M. Boehnke, "Joint analysis is more efficient than replication-based analysis for two-stage genome-wide association studies," Nature Genetics, vol. 38, no. 2, pp. 209-213, 2006.

[32] T. Sugiura, Y. Kawaguchi, K. Goto et al., "Positive association between STAT4 polymorphisms, and polymyositis/ dermatomyositis in a Japanese population," Annals of the Rheumatic Diseases, vol. 71, no. 10, pp. 1646-1650, 2012.

[33] S. Chen, Q. Wang, Z. Wu et al., "Genetic association study of TNFAIP3, IFIH1, IRF5 polymorphisms with polymyositis/dermatomyositis in Chinese Han population," PLoS ONE, vol. 9, no. 10, Article ID el10044, 2014.

[34] J. Freudenberg, P. Gregersen, and W. Li, "Enrichment of genetic variants for rheumatoid arthritis within T-cell and NK-cell enhancer regions," Molecular Medicine, vol. 21, pp. 180-184, 2015.

[35] I. Alloza, D. Otaegui, A. L. De Lapuente et al., "ANKRD55 and DHCR7 are novel multiple sclerosis risk loci," Genes and Immunity, vol. 13, no. 3, pp. 253-257, 2012.

[36] I. Gramaglia, A. D. Weinberg, M. Lemon, and M. Croft, "Ox-40 ligand: a potent costimulatory molecule for sustaining primary CD4 T cell responses," Journal of Immunology, vol. 161, no. 12, pp. 6510-6517, 1998. 
[37] K. H. Chua, Y. Y. Ooh, and H. C. Chai, “TNFSF4 polymorphisms are associated with systemic lupus erythematosus in the Malaysian population," International Journal of Immunogenetics, vol. 43, no. 5, pp. 303-309, 2016.

[38] Y. K. Chang, W. Yang, M. Zhao et al., "Association of BANK1 and TNFSF4 with systemic lupus erythematosus in Hong Kong Chinese," Genes and Immunity, vol. 10, no. 5, pp. 414-420, 2009.

[39] P. Gourh, F. C. Arnett, F. K. Tan et al., "Association of TNFSF4 (OX40L) polymorphisms with susceptibility to systemic sclerosis," Annals of the Rheumatic Diseases, vol. 69, no. 3, pp. 550-555, 2010. 


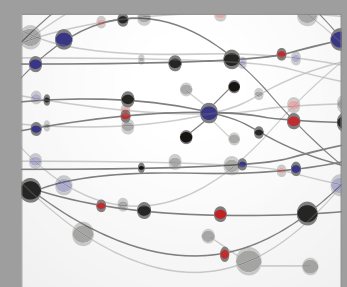

The Scientific World Journal
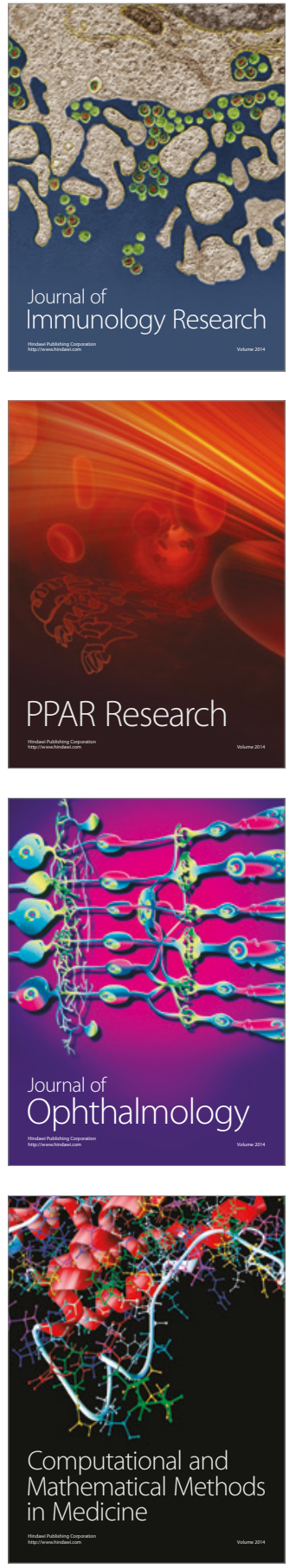

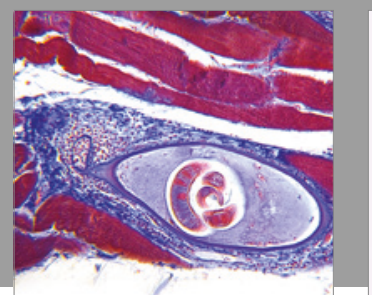

Gastroenterology Research and Practice
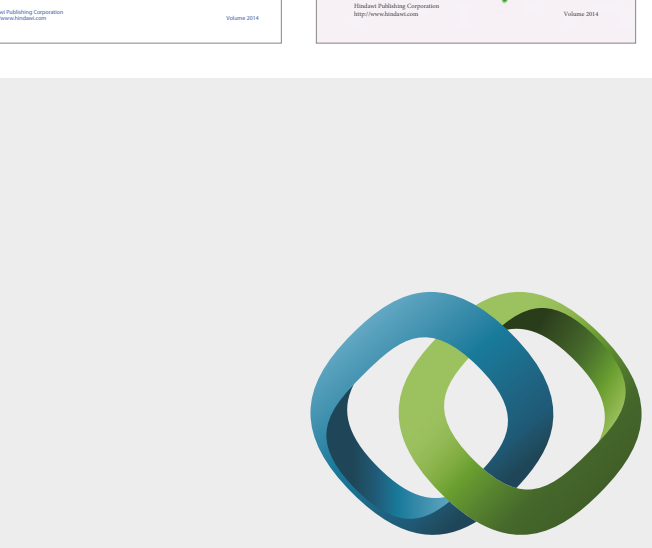

\section{Hindawi}

Submit your manuscripts at

https://www.hindawi.com
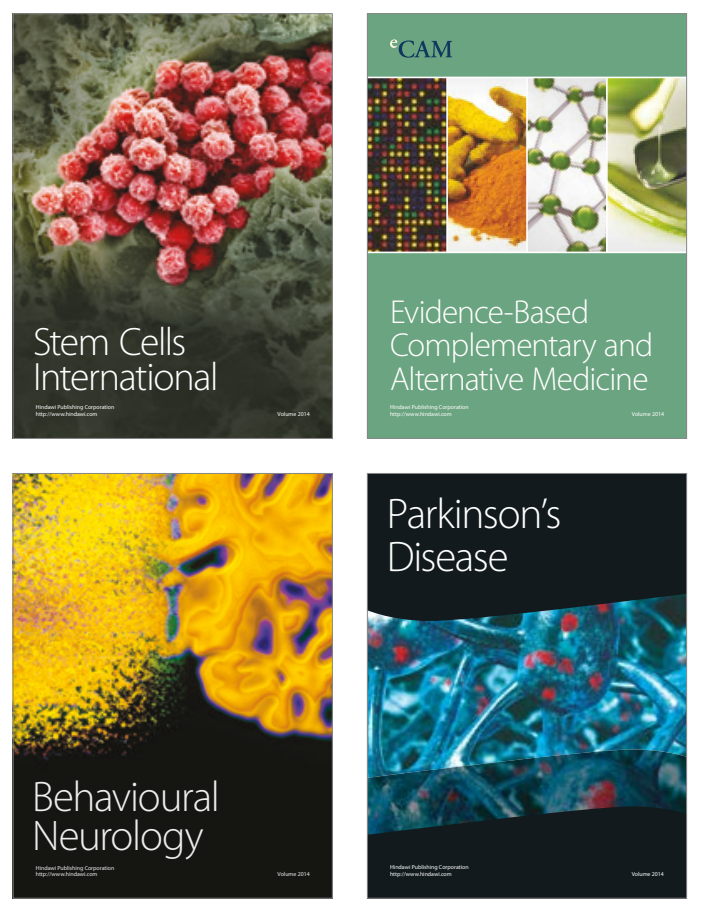
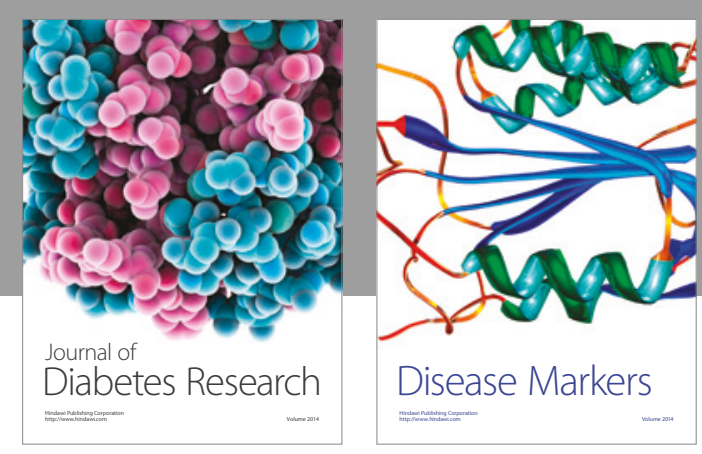

Disease Markers
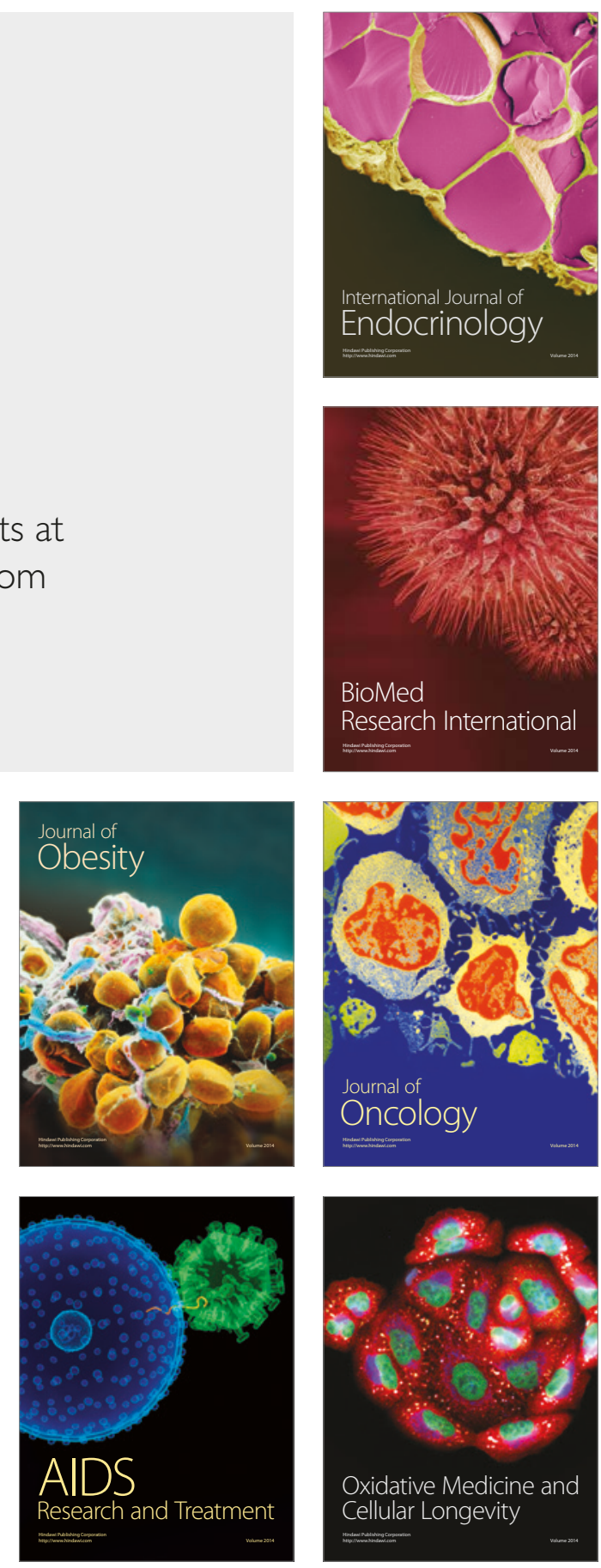